\title{
AUTOCUIDADO E PLANTAS MEDICINAIS - (DES) CONHECIMENTO DOS PROFISSIONAIS DE SAÚDE QUANTO AS PRÁTICAS CULTURAIS EM COMUNIDADES TRADICIONAIS
}

\section{SELF-CARE AND MEDICINAL PLANTS - (UN) KNOWLEDGE OF HEALTH PROFESSIONALS REGARDING CULTURAL PRACTICES IN TRADITIONAL COMMUNITIES}

\author{
Daniel Henrique Rodrigues Perdigão* \\ ORCID: https://orcid.org/0000-0002-6913-6956 \\ Victor Homero Barbosa** \\ ORCID: https://orcid.org/0000-0003-4442-6903 \\ Neudson Johnson Martinho*** \\ ORCID: https://orcid.org/0000-0001-9176-2729
}

\section{Resumo}

Trata-se de um estudo exploratório, descritivo e com abordagem qualitativa, recorte do projeto de extensão, com interface na pesquisa, intitulado "AUTOCUIDADO: Ações interprofissionais para promoção da saúde em comunidades tradicionais". Tem-se por objetivo apresentar os resultados obtidos com o desenvolvimento da primeira etapa do referido projeto. O mesmo foi executado no período de julho a dezembro de 2020, nos distritos de Nossa Senhora da Guia e do Aguaçú, no Estado de Mato Grosso, por bolsistas e voluntários do PET Saúde Interprofissionalidade. Participaram seis profissionais de saúde, com os quais foram realizadas três entrevistas focalizadas por meio do Google Meet. O projeto desvelou uma lacuna existente nos cursos da área de saúde e a urgente necessidade, de modo específico, da medicina, enfermagem e odontologia repensarem seus currículos visando à formação dos futuros profissionais com domínio cognitivo e técnico inerentes às práticas culturais de autocuidado à saúde em comunidades tradicionais, de forma peculiar, com o uso de plantas medicinais.

Palavras-chave: Autocuidado; Educação em saúde; Plantas medicinais.

Data recebimento: $26 / 01 / 2021$

Data de aceite: $23 / 04 / 2021$

\begin{abstract}
It is a qualitative, exploratory and descriptive study, part of the extension project with an interface in the research entitled "SELF-CARE: Interprofessional actions to promote health in traditional communities". It aims to present the results obtained with the development of the first stage of that project. It was carried out from July to December 2020, by scholarship students and volunteers from PET Health Interprofessional, in the districts of Nossa Senhora da Guia and Aguaçú, located in the State of Mato Grosso, Brazil. Six health professionals participated in three focused interviews conducted through Google Meet. The project unveiled a gap in health courses and the urgent need for Medicine, Nursing and Dentistry courses to rethink their curriculum in order to provide students cognitive and technical knowledge related to self-care cultural practices of traditional communities regarding the use of medicinal plants.
\end{abstract}

Keywords: Self-care; Health education; Medicinal plants.

\footnotetext{
* Aluno de Graduação da Universidade Federal de Mato Grosso (UFMT), Cuiabá - MT, Brasil. E-mail: delhenri98@gmail.com ** Aluno de Graduação da Universidade Federal de Mato Grosso (UFMT), Cuiabá - MT, Brasil. E-mail: victor.homero.barbosa@gmail.com.

*** Professor da Universidade Federal de Mato Grosso (UFMT), Cuiabá - MT, Brasil. E- mail: neudsonjm@hotmail.com
} 


\section{Introdução}

Práticas de cuidados com a saúde desenvolvidas por povos pertencentes a comunidades tradicionais e como intercambiar saberes acadêmicos com os mesmos durante a assistência profissional, ainda é algo desconhecido pela maioria dos profissionais de saúde. Esse desconhecimento, oriundo da ausência de abordagem sobre o assunto durante a formação acadêmica, se une à inexistência de políticas estruturantes por parte dos sistemas locais de saúde (SILOS).

Essa lacuna identificada na formação profissional na área da saúde, com reverberação nos serviços, pode ter consequências danosas nos cuidados à saúde desses povos específicos, tendo em vista que atitudes de desrespeito para com as práticas culturais de autocuidado com a saúde por eles desenvolvidas podem afastá-los dos serviços de saúde e/ou levá-los a não aderirem às terapêuticas prescritas e necessárias.

Frente a esse quadro evidenciado durante as práticas desenvolvidas pelo Programa Tutorial de Educação pelo Trabalho (PET) Saúde Interprofissionalidade Comunidades Tradicionais, foi elaborado o projeto de extensão, com interface na pesquisa, intitulado: "AUTOCUIDADO: Ações interprofissionais para promoção da saúde em comunidades tradicionais", cadastrado no sistema SIEX da Universidade Federal de Mato Grosso (UFMT) sob protocolo $\mathrm{n}^{\mathrm{o}} 240420201118441651$ e aprovado pelo Comitê de Ética em Pesquisa / Saúde/UFMT sob o parecer n ${ }^{\circ}$ 4.172.041/2020.

O referido projeto visou a identificar, neste período de pandemia de COVID-19, as ações de autocuidado que as comunidades tradicionais executavam e, a partir dessa identificação, ocorreu a realização de ações de educação em saúde como suporte para o uso seguro de tais práticas culturais.

Importante salientarmos o que são consideradas comunidades tradicionais. São povos indígenas; quilombolas, agroextrativistas, seringueiros, quebradeiras de coco babaçu, pescadores artesanais e caiçaras, raizeiros, vazanteiros, pantaneiros, ciganos, pomeranos, comunidades de terreiro, fundos de pasto, faxinais e ribeirinho. Esses povos têm características singulares que expressam sua identidade cultural, socioeconômica e religiosa, muitas vezes, por intermédio dos elementos da natureza (BRASIL, 2007; NETO et al., 2020; CARVALHO; MACEDO, 2018).

Cada povo tradicional traz consigo saberes e fazeres culturais adquiridos por seus ancestrais, não sendo diferente o autocuidado com a saúde individual e do coletivo, o qual se caracteriza por ações cotidianas imbricadas por crenças religiosas e com uso de elementos da natureza, particularmente, a utilização de plantas medicinais cultivadas na própria comunidade. 
O autocuidado pode ser definido como ações praticadas pelo próprio indivíduo, por uma família, grupo ou comunidade com o intuito de preservar e melhorar a saúde individual e/ou do coletivo, envolvendo o cuidado com os alimentos, o meio ambiente, o próprio corpo, sendo provenientes do aprendizado ancestral, de profissionais da saúde ou de membros da comunidade (SILVA et al., 2008).

Neste artigo, objetivamos apresentar os resultados obtidos com o desenvolvimento da primeira etapa do projeto de extensão supracitado, considerando que sua realização se revestiu de relevância social e acadêmica por possibilitar reflexões propositivas, tanto na dimensão da formação acadêmica quanto dos serviços de saúde, em relação ao conhecimento dos profissionais sobre saberes e fazeres culturais em saúde das comunidades tradicionais.

\section{Metodologia}

O projeto de extensão, com interface na pesquisa, "AUTOCUIDADO: Ações interprofissionais para promoção da saúde em comunidades tradicionais" foi desenvolvido por um bolsista de extensão e voluntários do PET Saúde Interprofissionalidade Comunidades Tradicionais da UFMT, nas Comunidades de Nossa Senhora do Livramento (cerca de $40 \mathrm{~km}$ de distância da capital Cuiabá) e do Aguaçú (cerca de $55 \mathrm{~km}$ de distância da capital), ambas são áreas rurais e fazem parte da baixada cuiabana, em Mato Grosso.

A execução do projeto foi dividida em duas etapas:

- $1^{\text {a }}$ etapa: caracterizou -se pelo levantamento junto aos profissionais das unidades de saúde da família das regiões lócus do projeto quanto ao nível de conhecimento destes em relação às práticas culturais de autocuidado com a saúde desenvolvidas pelas famílias. Essa etapa ocorreu no período compreendido de julho a dezembro de 2020. Em decorrência da pandemia de COVID-19, foi totalmente executada de maneira remota (on-line) por meio da plataforma Google Meet. Foram realizadas reuniões virtuais com os profissionais e entrevistas focalizadas com roteiro pré-determinado. Portanto, este estudo é um recorte dos resultados obtidos nessa primeira etapa de execução do projeto; 
- 2a etapa: prevista para ser efetivada no período de janeiro a julho de 2021 , a depender da evolução no declínio da pandemia e contará com o desenvolvimento de ações de educação em saúde junto aos profissionais e às comunidades, visando a realizar processos educativos quanto ao uso seguro e correto das práticas identificadas e estimular o intercâmbio entre o saber técnico e a educação popular em saúde.

Este estudo é do tipo exploratório, descritivo, com abordagem qualitativa, pois busca tornar mais explícito o problema investigado na tentativa de aprofundar as ideias sobre o objeto de investigação, delineando as características dos fenômenos identificados com descrições e observações emergentes da subjetividade dos participantes, tendo como propósito desvelar uma realidade significada nos relatos (SAMPIERI; COLLADO \& LÚCIO, 2006; GIL, 2011).

Foram estabelecidos os seguintes critérios de inclusão e exclusão para seleção dos participantes:

Inclusão:

- Ser profissional de saúde e estar atuando na unidade de saúde da família da comunidade locus do estudo;

- Aceitar participar do estudo assinando o Termo de Consentimento Livre e Esclarecido (TCLE).

Exclusão:

- Estar ausente do serviço por licença, atestado médico, férias ou outro motivo;

- Não concordar em participar do estudo.

Os profissionais de saúde participantes desse projeto foram: duas (02) enfermeiras, (01) médico e (02) odontólogos, todos eram preceptores do PET Saúde Interprofissionalidade, com exceção de um (01) odontólogo. Neste trabalho, preservando o anonimato da identificação, eles estão caracterizados com siglas, sendo EG (enfermeira da Guia), OG e MG (odontólogo e médico da Guia), assim como EA e OA (enfermeira e odontólogo do Aguaçú).

Para coleta de dados foi utilizado um roteiro subsidiador para realização de três entrevistas focalizadas por meio do Google Meet. Os dias e horários das entrevistas foram previamente acordados com os participantes. A entrevista focalizada, como o próprio nome aponta, foca em um tema específico, podendo o entrevistado falar livremente sobre o assunto, porém, o entrevistador deve ter o cuidado de sempre retomar o objetivo da mesma, não permitindo que o entrevistado se desvie do assunto em questão. Esse tipo de entrevista tem por objetivo explorar a fundo alguma experiência vivida em condições precisas, em que se deseja investigar algum fenômeno (GIL, 2011). 
Os dados coletados foram sistematizados por meio da técnica de análise temática e processo de categorização, segundo Bardin (2011), além da triangulação com a literatura e observações realizadas durante as rodas de conversa.

Esse processo de sistematização e análise dos fenômenos apreendidos nas falas dos participantes foi executado obedecendo as seguintes etapas:

$1^{\text {a }}$. Transcrição das falas;

$2^{\text {a }}$. Pré-análise: leitura flutuante dos discursos transcritos. A partir dessa leitura, foram identificadas as possíveis categorias;

3a. Realização de leituras exaustivas dos conteúdos das falas agrupadas por homogeneidade de sentidos para posterior organização dos conteúdos e agrupamento em grandes categorias;

$4^{\text {a }}$. Análises dos conteúdos das falas agrupadas por categorias, identificando unidades de significados, procedendo-se aos agrupamentos finais;

$5^{\text {a }}$. Análise e compreensão dos fenômenos identificados, descrevendo as significâncias (sentidos) dos mesmos.

\section{Resultados - Compreendendo os fenômenos desvelados nas falas}

\section{Categoria 01 - Visita às comunidades tradicionais: frequência e dificuldades.}

Ano passado nós realizamos visitas com maior frequência, mas esse ano (2020), elas não estão ocorrendo mais como rotina, porque a Secretaria Municipal de Saúde não está disponibilizando carro para transporte (EG).

De forma geral, nossas visitas estão ocorrendo apenas sobre demanda [...] (MG).

Nós só estamos realizando visitas em casos de emergência, como a Secretaria não tem disponibilizado transporte, nós vamos com ambulância daqui, com carro nosso mesmo (EA).

Nessa categoria, evidenciamos que os profissionais realizam poucas visitas domiciliares nas comunidades tradicionais, sendo a ausência de transporte para realizá-las o fator determinante apontado por eles.

Cruz e Bourget (2010) enfatizam que a não realização de visitas domiciliares possui consequências danosas na relação profissional com a comunidade. Assim, o pouco ou a inexistência desse contato do profissional com a comunidade pode resultar em medo, receio e pouca confiança da população em poder se entregar aos cuidados profissionais. Ademais, sem 
esse vínculo fortalecido, ocorre o problema da baixa procura das comunidades pelos serviços básicos de saúde, o que culmina com riscos para a saúde da população.

O contato com as comunidades é intrínseco à prática do autocuidado, pois esse favorece a troca de conhecimentos, estabelece vínculos, criando confiança entre a população e os profissionais, possibilitando melhor entendimento sobre a cultura local e assim viabiliza uma prática ampliada de saúde (KRIEGER et al, 2009; ROSÁRIO et al, 2020).

\section{Categoria 2 - Práticas culturais de autocuidado - (des)conhecimentos e (des)crenças}

Vi muitas mulheres da comunidade usarem o chá da folha de algodão para regular a menstruação, mas tem algumas plantas que eu fico meio desconfiada. Eu só acredito hoje na eficácia de algumas plantas pela convivência com os moradores daqui, porque eu nunca aprendi sobre esse assunto na faculdade (EG).

Eu não acreditava muito no uso das plantas medicinais para tratar doenças, mas, quanto mais eu convivia com a comunidade, mais eu observava os moradores recomendando, uns aos outros, algum tipo de erva para dor de cabeça, por exemplo. Na faculdade, isso não é valorizado (EA).

Olha, eu não acredito cem por cento na eficácia delas, porque existem diversas condições e contextos para eu considerá-las válidas [...] (MG).

[...] muitas mulheres se referem ao uso de rezas, benzeções, raizes e outras coisas. Acredito em parte por ter visto muitas comprovações nas práticas culturais desenvolvida. (OA).

É explícito nas falas dos profissionais a existência do desconhecimento e consequentemente descrença nas práticas culturais de autocuidado desenvolvidas pelas famílias das comunidades tradicionais. Alguns chegam a relatar alguma crença em plantas medicinais a partir da convivência com a comunidade, entretanto, deixam nas entrelinhas a falta de um conhecimento mais sólido que não obtiveram durante sua formação acadêmica profissional.

Segundo a teoria da aprendizagem experimental, formulada por David Kolb (1984), o conhecimento experimental possui utilidade para o desenvolvimento profissional à medida que ele é comparado, analisado, confrontado e revisado com o conhecimento teórico (PIMENTEL, 2007).

Nesse sentido, notamos por meio dos relatos que existe uma sobreposição do conhecimento empírico sobre o teórico, o que pode ser prejudicial na aprendizagem e prática dos profissionais, visto a necessidade de haver bases teóricas sólidas e estruturadas para orientar o seu próprio aprendizado e posterior repasse à comunidade. 
Esse fenômeno identificado nos leva a inferir que apesar de existirem vários estudos e pesquisas sobre uso de plantas medicinais e outras práticas culturais de cuidado à saúde, nos cursos da área da saúde ainda existe essa lacuna na formação. Durante a mesma não se ensina aos futuros profissionais as práticas culturais em saúde, como o uso de plantas medicinais pelas famílias, práticas inerentes à cultura do nosso país, rico em fauna, flora e culturas diversas.

Os profissionais da área da saúde possuem importante papel estimulador e indutor de práticas de autocuidado nas comunidades, principalmente no que diz respeito a produzirem e consumirem plantas medicinais de forma segura; todavia, se esses profissionais não possuem conhecimentos sobre fitoterapia, o papel educativo fica a desejar, gerando impasses na implementação do autocuidado e da promoção da saúde, além de perigos quanto ao uso incorreto, seja de plantas medicinais ou de outras práticas culturais em saúde (SANTOS et al., 2016).

Neto et al (2020) destacam que alguns elementos são intrínsecos ao estilo de vida e cultura dessas comunidades tradicionais, como a utilização de plantas medicinais, as quais podem ser manejadas em favor do autocuidado e bem-estar, considerando o potencial destas como meio de terapia complementar para a manutenção da saúde.

A Política e Programa Nacional de Plantas Medicinais e Fitoterápicos (PPNPMF) ressalta que muitas populações possuem pouco acesso aos serviços de saúde e por isso recorrem ao conhecimento popular de plantas medicinais para sanar suas necessidades curativas (BRASIL, 2016).

\section{Categoria 3 - Plantas medicinais: (des) conhecimentos.}

Sobre plantio e colheita eu não sei nada! O que eu sei é pelo o que os pacientes trazem, como o algodão para inflamação, mas não sei como ela atua de fato no organismo (EG).

A única coisa que eu sei é que a folha de batata serve para desinchar, a vassourinha é cicatrizante, cidreira é calmante e boldo é bom para o estômago (OG).

O conhecimento que eu tenho é muito escasso, só sei sobre o uso e recomendações de algumas plantas que a própria comunidade compartilha (MG).

Não conheço tanto as plantas, mas descobri quais têm sido mais recomendadas na pandemia: melão-de-são-caetano, pina, além do chá de amora para emagrecer. O povo tem feito muito chá, para eles tudo que é quente mata o coronavirus (EA).

Essa categoria corrobora as demais acima citadas, reafirmando a escassez de conhecimentos dos profissionais de saúde sobre plantas medicinais, desde a sua produção, 
manejo, armazenamento, conhecimento integral sobre as contraindicações, interações com alimentos e medicamentos alopáticos e nível de toxicidade.

Tal desconhecimento dos profissionais de saúde sobre as plantas medicinais e consequentemente pouca importância dada quanto às que são utilizadas pelas comunidades é algo perigoso sob vários aspectos, podendo ser considerado um problema de saúde pública. É importante considerarmos que esses profissionais não saberão orientar a comunidade sobre o uso correto dessas plantas enquanto terapêuticas complementares, o que poderá ter implicações danosas à saúde individual e coletiva na comunidade. Isso pode ocorrer a curto, médio e longo prazo, indo do mais simples ao mais complexo evento adverso, como uma hepatotoxicidade grave ou mesmo envenenamentos.

Os cursos da área de saúde, especificamente os de medicina, enfermagem e odontologia, precisam repensar a formação e melhor preparar os futuros profissionais sobre as plantas medicinais existentes no Brasil e já comprovadas quanto aos seus efeitos farmacológicos para que estes saibam orientar a comunidade sobre como utilizá-las de maneira correta e segura (ZENI et al, 2017; SANTOS et al, 2016).

\section{Categoria 4 - Correto ou errado? Uso de plantas medicinais pela comunidade}

É dificil falar, pois a comunidade entende como o certo. E aí precisamos estudar pra saber se realmente a planta tem a sua eficácia ou não. Tudo que se fala aqui é chá ou planta, a comunidade não entende que nada faz mal, para eles tudo faz bem. É dificil dizer o que é correto ou errado. Às vezes eles deixam de usar a medicação para usar as plantas medicinais, pois eles acreditam na maior eficácia delas (MG).

Eu não me vejo com propriedade para dizer com total certeza o que é indevido ou não, porque eu não sei bem como cada planta deve ser utilizada, não tive esse preparo na faculdade(EG).

Isso é complicado, porque não sei qual a dosagem certa, quantas vezes eles deviam utilizar ou de que forma armazenar. Mas, o que mais me preocupa é quando eles deixam de utilizar a medicação que recomendamos para fazer uso das plantas (EA).

Não recomendo o uso de várias plantas, porque aqui tem a mentalidade do bochecho e na verdade é preciso o coágulo após intervenção cirúrgica odontológica. Esse bochecho prejudica o processo de cicatrização, porque remove o coágulo e aumenta o sangramento. No geral, tem plantas que têm o poder e eficácia para dor de cabeça, serem diuréticas, mas, a parte mecânica do bochecho remove coágulos. Em odontologia isso não é indicado (OG).

Observamos nesses relatos a insegurança dos profissionais em identificar se o uso das plantas medicinais está sendo correto ou errado, reafirmando o déficit de conhecimento já abordado em outros momentos neste estudo. 
Santos et al (2016) enfatizam que eventos adversos como sangramentos, náuseas, vômitos ou mesmo desfechos tóxicos ao utilizar plantas medicinais de diversas formas (chás, bochechos, gargarejos, pomadas, cremes ou outros) poderiam ser evitados ou amenizados se os profissionais tivessem conhecimentos sobre essas terapêuticas. Assim, cresce a necessidade das universidades, secretarias municipais e estaduais de saúde investirem em capacitações em fitoterapia para os profissionais de saúde, de modo específico para os que atuam em unidades básicas de saúde da família.

\section{Considerações finais}

A construção social da saúde perpassa por processos educativos dialógicos, mas esses processos não ocorrerão se os profissionais de saúde não tiverem conhecimentos sólidos quanto aos assuntos a serem dialogados. Isso é condição para que possa ocorrer uma circularidade de ensinamentos e aprendizagens, onde quem ensina aprende e quem aprende ensina, numa troca de saberes técnicos e populares a partir das experiências vividas e seus sentidos.

As comunidades tradicionais precisam ter suas práticas culturais de cuidado à saúde valorizadas e estimuladas, porém de forma segura e equânime, sendo a relação dialógica entre profissionais da saúde e indivíduo, família e comunidade, uma condição sine qua non nesse processo. Mas não haverá esse diálogo respeitoso e educativo se os profissionais da saúde desconhecem tais práticas em suas dimensões biopsicossociais.

Nesse contexto, o projeto desvelou uma lacuna existente na formação dos profissionais de saúde, de modo específico nos cursos de medicina, enfermagem e odontologia, que devem repensar seus currículos visando a proporcionar competências e habilidades na dimensão cognitiva e técnica inerentes às práticas culturais de autocuidado à saúde em comunidades tradicionais, de forma peculiar, quanto ao uso de plantas medicinais.

A realização de projetos de extensão, com interface na pesquisa, tem demonstrado ser uma estratégia eficaz na integração ensino-serviço-comunidade e o Pet Saúde Interprofissionalidade reverbera essa importância ao possibilitar a aproximação da academia com os serviços e as comunidades tradicionais, trazendo à tona reflexões críticas-propositivas quanto ao ensino e à atuação profissional de forma mais eficaz no cuidado à saúde desses povos específicos. 
Concluímos que o desenvolvimento de outros projetos dessa natureza é de suma importância nas comunidades tradicionais, os quais devem ser inseridos na curricularização da extensão dos cursos da área de saúde nas instituições de ensino superior do Brasil, para que as práticas culturais de autocuidado sejam pesquisadas, reconhecidas, valorizadas e a educação em saúde aconteça efetivamente nos serviços de saúde.

\section{Referências}

BARDIN, L. Análise de conteúdo. São Paulo: Edições 70, 2011.

BRASIL. Presidência da República. Decreto n 6040, de 7 de fevereiro de 2007. Diário Oficial da União, s. 1, p.316, Brasília-DF, 2007.

. Política Nacional e Programa Nacional de Plantas Medicinais e Fitoterápicos.

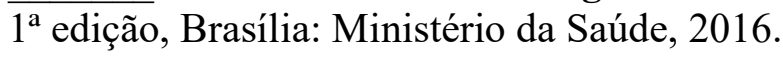

CARVALHO, A. V.; MACEDO, J. P. (2018). Povos e comunidades tradicionais: revisão sistemática da produção de conhecimento em Psicologia. Revista Psicologia: Teoria e Prática, 20(3), 180-197.

CRUZ, M. M.; BOURGET, M. M. M (2010). A Visita Domiciliária na Estratégia de Saúde da Família: conhecendo as percepções das famílias. Saúde e Sociedade, 19(3), 605-613.

GIL, A. C. Métodos e técnicas de pesquisa social. 6.ed. São Paulo: Atlas, 2011.

KRIEGER, J.; TAKARO, T. K.; SONG, L.; BEAUDET, N.; EDWARDS, K. (2009). The Seattle-King County Healthy Homes II Project: A Randomized Controlled Trial of Asthma Self-management Support Comparing Clinic-Based Nurses and In-Home Community Health Workers. Archives of Pediatrics \& Adolescent Medicine, 163(2), 141-149.

NETO, C. F. A.; VALADARES, G. V.; COSTA, L. S. (2020). Revisão Integrativa sobre a saúde da comunidade tradicional: Reflexões ecológicas. Revista Saúde e Meio Ambiente RESMA, 10(1), 82-94.

PIMENTEL, A. (2007). A teoria da aprendizagem experiencial como alicerce de estudos sobre desenvolvimento profissional. Estudos de Psicologia, 12(2), 159-168 
ROSÁRIO, C. A.; BAPTISTA, T. W. F.; MATTA, G. C. (2020). Sentidos da universalidade na VIII Conferência Nacional de Saúde: entre o conceito ampliado de saúde e a ampliação do acesso a serviços de saúde. Rev. Saúde em debate, 44 (124), 17-31.

SAMPIERI, R. H.; COLLADO, C. F.; LUCIO, P. B. Definição da Pesquisa a ser realizada: exploratória, descritiva, co - relacional ou explicativa. In: SAMPIERI, R. H.; COLLADO, C. F.; LUCIO, P. B. Metodologia de pesquisa. $3^{\circ}$ ed. São Paulo: McGraw-Hill, 2006.

SANTOS, J. A. A.; SANTOS, E. C. B.; MAGNATA, S. S. L. P.; GARCIA, J. E.; MARTINS, R. D. (2016). Diagnóstico e educação em saúde no uso de plantas medicinais: relato de experiência. Revista Ciência em Extensão, 12(4), 183-196.

SILVA, I. J.; OLIVEIRA, M. F. V.; SILVA, S. E. D.; POLARO, S. H. I.; RADUNZ, V.; SANTOS, E. K. A.; SANTANA, M. E. (2008). Cuidado, autocuidado e cuidado de si: uma compreensão paradigmática para o cuidado em enfermagem. Revista da Escola de Enfermagem da USP, 43(3), 697-703.

ZENI, A. L. B.; PARISOTTO, A. V.; MATTOS, G.; HELENA, E. T. S. (2017). Utilização de plantas medicinais como remédio caseiro na Atenção Primária em Blumenau, Santa Catarina, Brasil. Revista Ciência \& Saúde Coletiva, 22(8), 2703-2712. 